\title{
CLOSED COUNTABLY GENERATED STRUCTURES IN $C(X)$
}

BY

B. ROTH

ABSTRACT. Let $C(X)$ be the space of continuous real or complex valued functions on a compact space $X$ with the sup norm topology. In the present paper, the subalgebras, vector lattices, and vector lattice ideals of $C(X)$ which are closed and countably generated are characterized.

1. Introduction. M. H. Stone, in his pioneering work [2], characterized the set of functions in $C(X)$ generated by a subset $S$ of $C(X)$ via the application of specified algebraic operations and uniform passage to the limit. When is the set of functions generated by $S$ using only the algebraic operations already closed under uniform passage to the limit, that is, when is the algebraic structure generated by $S$ (by which we mean the smallest algebraic structure containing $S$ ) closed in the sup norm topology? Of course Stone's results provide one answer to this question, but by restricting attention to finite or, more generally, countable subsets of $C(X)$, much more can be said.

For the lattice operations in real $C(X)$, the structures generated by any finite subset of $C(X)$ are closed. Specifically, every finitely generated lattice in $C(X)$ is closed since the lattice generated by any finite subset of $C(X)$ is itself finite and every finitely generated lattice ideal in $C(X)$ is closed since the lattice ideal generated by $f_{1}, \cdots, f_{n} \in C(X)$ is

$$
\left\{f \in C(X): f \leq \max \left(f_{1}, \cdots, f_{n}\right)\right\} \text {. }
$$

However, when we turn our attention to the algebra or vector lattice structure of $C(X)$, the situation changes dramatically and we find that it is rather unusual for even a countably generated structure to be closed. For example, the scarcity of closed countably generated algebra ideals is pointed out by the following theorem, the nontrivial part of which is due to L. Gillman [1, Theorem 5.2, pp. 663-664].

Presented to the Society, January 18, 1972 under the title Closed finitely generated structures in $C(X)$; received by the editors February 4, 1972.

AMS (MOS) subject classifications (1970). Primary 46E05, 46E25; Secondary 46A40, $46 \mathrm{~J} 10$.

Key words and phrases. Spaces of continuous functions, subalgebras of continuous functions, vector lattices of continuous functions, countably generated subalgebras, countably generated vector lattices, countably generated vector lattice ideals. 
Theorem 1. Let I be an algebra ideal in real or complex $C(X)$. The following are equivalent:

(a) $I$ is closed and countably generated;

(b) $Z(I)$ is open in $X$;

(c) $I$ is closed and generated by an idempotent.

Since $Z(I)$ is always closed in $X$, there are no proper closed countably generated algebra ideals in $C(X)$ if $X$ is connected. (Throughout the paper, for $S \subset$ $C(X)$ we let

$$
Z(S)=\bigcap\{Z(f): f \in S\}
$$

where $Z(f)$ denotes the set of zeros of $f$.

2. Subalgebras. If $A$ is a subalgebra of $C(X)$, we define the equivalence relation $\sim_{A}$ on $X$ by $x \sim_{A} y$ if and only if $f(x)=f(y)$ for all $f \in A$. Then $\operatorname{cl}(A)=$ $\left\{f \in C(X): f=0\right.$ on $Z(A)$ and $f(x)=f(y)$ whenever $\left.x \sim_{A} y\right\}$ by the Stone-Weierstrass theorem [2, Theorem 5, p. 46].

Theorem 2. Let $A$ be a subalgebra of real or complex $C(X)$. The following are equivalent:

(a) $A$ is closed and countably generated;

(b) A is finite dimensional;

(c) there are only finitely many distinct equivalence classes with respect to $\sim_{A}$;

(d) A is closed, singly generated, and selfadjoint.

Proof. Let $F$ denote the scalar field.

(a) implies (b) is an immediate consequence of the well-known fact that there are no Banach spaces of countably infinite Hamel dimension.

(b) implies (c). Suppose $A$ is finite dimensional, say $f_{1}, \cdots, f_{n}$ is a basis for $A$. We show that there are not more than $m=n+1$ distinct equivalence classes with respect to $\sim_{A}$. For suppose there are more than $m$ distinct equivalence classes. If $Z(A) \neq \varnothing, Z(A)$ is one of the equivalence classes. Thus there are $m$ equivalence classes distinct from $Z(A)$, say $\left[x_{1}\right], \cdots,\left[x_{m}\right]$. The subalgebra

$$
A^{\prime}=\left\{\left(f\left(x_{1}\right), \cdots, f\left(x_{m}\right)\right): f \in A\right\}
$$

of $F^{m}$ is separating and without zeros. Since every subalgebra of $F^{m}$ is selfadjoint, we can conclude that $A^{\prime}=F^{m}$ by the Stone-Weierstrass theorem. Therefore the $n$ vectors $\left(f_{i}\left(x_{1}\right), \cdots, f_{i}\left(x_{m}\right)\right), 1 \leq i \leq n$, span $F^{m}$ which is impossible. Hence there are not more than $m=n+1$ distinct equivalence classes with respect to $\sim_{A}$. In fact, having established that there are only finitely many distinct equivalence classes, it is not hard to show that there are exactly $n$ distinct equivalence classes if $Z(A)=\varnothing$ and $n+1$ if $Z(A) \neq \varnothing$. 
(c) implies (d). Suppose there are $n$ distinct equivalence classes with respect to $\sim_{A}$, say $\left[x_{1}\right], \cdots,\left[x_{n}\right]$. Consider the subalgebra

$$
A^{\prime}=\left\{\left(f\left(x_{1}\right), \ldots, f\left(x_{n}\right)\right): f \in A\right\}
$$

of $F^{n}$. Then

$$
A^{\prime}=\left\{\left(y_{1}, \cdots, y_{n}\right): y_{i}=0 \text { if }\left[x_{i}\right]=Z(A)\right\}
$$

by the Stone-Weierstrass theorem. The algebra homomorphism $f \rightarrow\left(f\left(x_{1}\right), \ldots\right.$, $\left.f\left(x_{n}\right)\right)$ of

$$
\left\{f \in C(X): f=0 \text { on } Z(A) \text { and } f(x)=f(y) \text { whenever } x \sim_{A} y\right\}
$$

onto $A^{\prime}$ is one-to-one. This implies that

$$
A=\left\{f \in C(X): f=0 \text { on } Z(A) \text { and } f(x)=f(y) \text { whenever } x \sim_{A} y\right\}
$$

and hence $A$ is closed and selfadjoint. Furthermore, since $A^{\prime}$ is generated by any $\left(y_{1}, \cdots, y_{n}\right) \in A^{\prime}$ with $y_{i} \neq y_{j}$ for $i \neq j$, we conclude that $A$ is singly generated.

Corollary. If $X$ is connected, then the only proper closed countably generated subalgebra of $C(X)$ is the subalgebra of constant functions.

Proof. Suppose $A$ is a proper closed countably generated subalgebra of $C(X)$. Since each equivalence class with respect to $\sim_{A}$ is closed and $X$ is connected, (c) implies that there is only one equivalence class. Thus $Z(A)=\varnothing$ since $A$ is a proper subalgebra and hence $A$ is the subalgebra of constant functions on $X$.

3. Vector lattices. Suppose $L$ is a vector lattice in real $C(X)$, that is, a linear subspace of $C(X)$ which is closed under the formation of absolute values. For $\lambda>0$ and $x, y \in X$, we define $x \lambda_{L} y$ if and only if $f(x)=\lambda f(y)$ for all $f \in L$. Then

$$
\operatorname{cl}(L)=\left\{f \in C(X): f(x)=\lambda f(y) \text { whenever } x \lambda_{L} y\right\}
$$

by Stone's theorem on vector lattices [2, Theorem 3, p. 39]. We define the equivalence relation $\sim_{L}$ on $X$ by $x \sim_{L} y$ if and only if there exists $\lambda>0$ with $x \lambda_{L} y$.

We begin with a discussion of countably generated vector lattices which will be used again in $\$ 4$ on vector lattice ideals. Suppose $L$ is the vector lattice generated by $\left\{f_{1}, f_{2}, \cdots\right\}$. For each $n$, let $V_{n}$ be the linear subspace of $C(X)$ spanned by $\left\{f_{i}\right\}_{1 \leq i \leq n}$. And for each $n$, let $L_{n}^{\prime}$ be the set of functions $f \in C(X)$ for which there exists a partition $X_{1}, \ldots, X_{k}$ of $X$ where $k \leq n$ such that for $1 \leq i \leq k$ :

(i) if $x \in X_{i}$, then the equivalence class $[x] \subset X_{i}$;

(ii) there exists $g_{i} \in V_{n}$ with $f\left|X_{i}=g_{i}\right| X_{i}$

Then it is a simple matter to verify that: 


$$
\begin{aligned}
& \text { if } f \in L_{n}^{\prime} \text { and } g \in L_{m}^{\prime} \text {, then } \alpha f+\beta g \in L_{n m}^{\prime} \text { for all } \alpha, \beta \in R \\
& \text { if } f \in L_{n}^{\prime} \text {, then }|f| \in L_{2 n}^{\prime} \text {. }
\end{aligned}
$$

Thus $L^{\prime}=\bigcup_{n} L_{n}^{\prime}$ is a vector lattice containing $\left\{f_{1}, f_{2}, \ldots\right\}$ and hence $L \subset L^{\prime}$ because $L$ is the smallest vector lattice containing $\left\{f_{1}, f_{2}, \ldots\right\}$. Moreover, $L^{\prime} C$ $\mathrm{cl}(L)$ by Stone's theorem on vector lattices.

Theorem 3. Let $L$ be a vector lattice in real $C(X)$. The following are equivalent:

(a) $L$ is closed and countably generated;

(b) there are only finitely many distinct equivalence classes with respect to $\sim_{L}$;

(c) $L$ is finite dimensional;

(d) $L$ is closed and finitely generated.

Proof. (a) implies (b). Suppose $L$ is closed and generated by $\left\{f_{1}, f_{2}, \cdots\right\}$. Consider $L^{\prime}=\bigcup_{n} L_{n}^{\prime}$ as described above. Then $L=L^{\prime}$ since $L$ is closed. We show that if there are infinitely many equivalence classes with respect to $\sim_{L}$, then each $L_{n}^{\prime}$ is nowhere dense in the complete metric space. $L$, contradicting the Baire category theorem.

To establish that $L_{n}^{\prime}$ is nowhere dense in $L$, it suffices to prove that for every $\epsilon>0$ there exists $b \in L-\operatorname{cl}\left(L_{n 2}^{\prime}\right)$ with $\|b\| \leq \epsilon$. For consider $f \in \operatorname{cl}\left(L_{n}^{\prime}\right)$ and $\epsilon>0$. Let $g=f+b$ where $b \in L \frac{n}{-} \operatorname{cl}\left(L_{n}^{\prime}{ }_{2}\right)$ and $\|b\| \leq \epsilon$. Then $\|f-g\| \leq \epsilon$ and, moreover, $g \in L-\mathrm{cl}\left(L_{n}^{\prime}\right)$. For suppose ${ }^{n} g \in \mathrm{cl}\left(L_{n}^{\prime}\right)$. Then there exist sequences $\left\{b_{k}\right\}$ and $\left\{g_{k}\right\}$ in $L_{n}^{\prime}$ with $b_{k} \rightarrow f$ and $g_{k} \rightarrow g$. Therefore $g_{k}-b_{k} \rightarrow g-f=b$ where $g_{k}-b_{k} \in L_{n 2}^{\prime}$ for all $k$ by (1). Hence $b \in \mathrm{cl}\left(L_{n 2}^{\prime}\right)$, contradicting our choice of $b$.

We now demonstrate that for every $n$ and every $\epsilon>0$ there exists $b \in L-$ $\mathrm{cl}\left(L_{n}^{\prime}\right)$ with $\|b\| \leq \epsilon$ and it is at this point that we utilize our assumption that there are infinitely many equivalence classes with respect to $\sim_{L}$. If $Z(L) \neq \varnothing, Z(L)$ is one of the equivalence classes. Let $m=n^{2}+1$ and choose $\left\{x_{i}\right\}_{1 \leq i \leq m} \subset X$ with $\left[x_{i}\right] \neq\left[x_{j}\right]$ for $i \neq j$ and $\left[x_{i}\right] \neq Z(L)$ for $1 \leq i \leq m$. An elementary linear dependence argument establishes the existence of $\left(a_{1}, \cdots, a_{m}\right) \in \mathfrak{R}^{m}$ such that no more than $n$ of the $m$ equations

$$
\begin{array}{ccc}
\lambda_{1} f_{1}\left(x_{1}\right)+\cdots+\lambda_{n} f_{n}\left(x_{1}\right)= & a_{1} \\
\vdots & \vdots & \vdots \\
\lambda_{1} f_{1}\left(x_{m}\right)+\cdots+\lambda_{n} f_{n}\left(x_{m}\right) & =a_{m}
\end{array}
$$

are simultaneously solvable. The vector lattice

$$
\left\{\left(f\left(x_{1}\right), \cdots, f\left(x_{m}\right)\right): f \in L\right\}
$$


equals $\mathfrak{R}^{m}$ by our choice of $\left\{x_{i}\right\}_{1 \leq i \leq m}$ and Stone's theorem on vector lattices. Thus there exists $b^{\prime} \in L$ with $b^{\prime}\left(x_{i}\right)=a_{i}$ for $1 \leq i \leq m$. Let $b=\epsilon b^{\prime} /\left\|b^{\prime}\right\|$. Then $b \in L,\|b\|=\epsilon$, and no more than $n$ of the $m$ equations

$$
\begin{aligned}
& \lambda_{1} f_{1}\left(x_{1}\right)+\cdots+\lambda_{n} f_{n}\left(x_{1}\right)=b\left(x_{1}\right) \\
& \lambda_{1} f_{1}\left(x_{m}\right)+\cdots+\lambda_{n} f_{n}\left(x_{m}\right)=b\left(x_{m}\right)
\end{aligned}
$$

are simultaneously solvable.

Finally, we show that $b \in L-\operatorname{cl}\left(L_{n}^{\prime}\right)$. Suppose $b \in \operatorname{cl}\left(L_{n}^{\prime}\right)$. Then there exists a sequence $\left\{b_{j}\right\}$ in $L_{n}^{\prime}$ with $b_{j} \rightarrow b$. Since each $b_{j} \in L_{n}^{\prime}$, there exists a partition of $X$ into $\leq n$ subsets for each $j$ by the definition of $L_{n}^{\prime}$. And some subset in each partition contains more than $n$ elements of $\left\{x_{i}\right\}_{1 \leq i \leq m}$ since $m=n^{2}+1$. Hence there exists a subset $X^{\prime}=\left\{x_{i_{1}}, \cdots, x_{i}\right\}$ of $\left\{x_{i}\right\}_{1 \leq i \leq m}$ where $l>n$ and a subsequence $\left\{b_{j_{k}}\right\}$ of $\left\{b_{j}\right\}$ such that for each $k, X^{\prime}$ is contained in some subset in the partition of $X$ corresponding to $b_{j_{k}}$. Therefore for each $k$ there exists $g_{k} \epsilon$ $V_{n}$ with $b_{j_{k}}\left|X^{\prime}=g_{k}\right| X^{\prime}$ by the definition of $L_{n}^{\prime}$. But the linear subspace

$$
V=\left\{\left(g\left(x_{i_{1}}\right), \cdots, g\left(x_{i_{l}}\right)\right): g \in V_{n}\right\}
$$

of $\mathbb{R}^{l}$ is closed and hence $\left(b\left(x_{i_{1}}\right), \cdots, b\left(x_{i_{l}}\right)\right) \in V$ because

$$
\left(g_{k}\left(x_{i_{1}}\right), \cdots, g_{k}\left(x_{i_{l}}\right)\right)=\left(b_{j_{k}}\left(x_{i_{1}}\right), \ldots, b_{j_{k}}\left(x_{i_{l}}\right)\right) \rightarrow\left(b\left(x_{i_{1}}\right), \ldots, b\left(x_{i_{l}}\right)\right) .
$$

Thus there exists $g=\lambda_{1} f_{1}+\cdots+\lambda_{n} f_{n} \in V_{n}$ with $\left(g\left(x_{i_{1}}\right), \cdots, g\left(x_{i_{l}}\right)\right)=\left(b\left(x_{i_{1}}\right), \cdots\right.$, $\left.b\left(x_{i}\right)\right)$ which means that $\lambda_{1}, \cdots, \lambda_{n}$ satisfies $l>n$ of the equations (2), a contradiction.

(b) implies (c). Suppose there are $n$ distinct nonzero equivalence classes with respect to $\sim_{L}$, say $\left[x_{1}\right], \cdots,\left[x_{n}\right]$ where $\left[x_{i}\right] \neq Z(L)$ for $1 \leq i \leq n$. The vector lattice

$$
\left\{\left(f\left(x_{1}\right), \ldots, f\left(x_{n}\right)\right): f \in L\right\}
$$

equals $\mathfrak{R}^{n}$ by Stone's theorem on vector lattices. Choose $f_{1}, \ldots, f_{n} \in L$ with $f_{i}\left(x_{j}\right)=\delta_{i j}$ for $1 \leq i, j<n$. Then the functions $\left\{f_{i}\right\}_{1 \leq i \leq n}$ form a basis for $L$ as is easily verified.

(c) implies (d) is immediate since if $\left\{f_{i}\right\}_{1 \leq i \leq n}$ is a basis for $L$, then the vector lattice generated by $\left\{f_{i}\right\}_{1 \leq i \leq n}$ is $L$.

Singly generated vector lattices in $C(X)$ have dimension at most two. Thus all singly generated vector lattices in $C(X)$ are closed but it is a simple matter to construct closed finitely generated vector lattices in $C(X)$ which are not singly generated. 
4. Vector lattice ideals. Suppose $I$ is a vector lattice ideal in real $C(X)$, that is, a vector lattice with the property that $g \in I$ whenever $f \in I$ and $|g| \leq|f|$. Then

$$
\mathrm{cl}(I)=\{f \in C(X): f=0 \text { on } Z(I)\}
$$

by Stone's theorem on vector lattice ideals [2, Theorem 7, p. 52] which is essentially due to Kakutani.

Theorem 4. Let I be a vector lattice ideal in real $C(X)$. The following are equivalent:

(a) I is closed and countably generated;

(b) $Z(I)$ is open in $X$;

(c) $I$ is closed and generated by an idempotent.

Proof. (a) implies (b). Suppose $I$ is closed and generated by $\left\{f_{1}, f_{2}, \ldots\right\}$. Let $L$ be the vector lattice generated by $\left\{f_{1}, f_{2}, \ldots\right\}$ and consider the vector lattice $L^{\prime}=\bigcup_{n} L_{n}^{\prime}$ constructed from $L$ in the manner described in $\$ 3$. Then

$$
I^{\prime}=\left\{g \in C(X):|g| \leq|f| \text { for some } f \in L^{\prime}\right\}
$$

is easily seen to be a vector lattice ideal containing $\left\{f_{1}, f_{2}, \ldots\right\}$ and thus $I \subset I^{\prime}$. Moreover, $I^{\prime}$ C I since

$$
I=\operatorname{cl}(I)=\{f \in C(X): f=0 \text { on } Z(I)\} .
$$

Hence $I=I^{\prime}$.

An argument similar to that employed by Gillman [1, Theorem 5.2, pp. 663-664] can now be used to show that $Z(I)$ is open in $X$. Clearly we may assume that each $\left\|f_{m}\right\| \leq 1$. Then

$$
b=\sum_{m}\left|f_{m}\right|^{1 / 2 / 2^{m}} \in C(X)
$$

and, furthermore, $b \in I$ since $b=0$ on $Z(I)=\bigcap_{m} Z\left(f_{m}\right)$. Thus there exists $f \in L^{\prime}$, say $f \in L_{n}^{\prime}$, with $|b| \leq|f|$. By the definition of $L_{n}^{\prime}$ (see $\S 3$ ), there exists a partition $X_{1}, \cdots, X_{k}$ of $X$ where $k \leq n$ such that for $1 \leq i \leq k$ there exists $g_{i} \in V_{n}$ with $f\left|X_{i}=g_{i}\right| X_{i}$. Since each $g_{i} \in V_{n}$, the linear subspace spanned by $\left\{f_{j}\right\}_{1 \leq j \leq n}$, we have

$$
g_{i}=\sum_{j=1}^{n} \lambda_{i j} f_{j}
$$

for $1 \leq i \leq k$. Choose $\lambda>0$ with

$$
\lambda \geq \max \left\{\left|\lambda_{i j}\right|: 1 \leq i \leq k, 1 \leq j \leq n\right\}
$$

Then we have

$$
b=|b| \leq|f| \leq \lambda\left(\left|f_{1}\right|+\cdots+\left|f_{n}\right|\right) .
$$


Since each $f_{i}$ vanishes on $Z(I)$, there is an open set $U$ containing $Z(I)$ such that $\left|f_{i}(x)\right|^{1 / 2}<1 / \lambda 2^{n}$ for all $x \in U$ and $1 \leq i \leq n$. Thus for $1 \leq i \leq n$ and $x \in U$, we have

$$
\lambda\left|f_{i}(x)\right| \leq\left|f_{i}(x)\right|^{1 / 2} / 2^{n}
$$

with strict inequality if $f_{i}(x) \neq 0$. But if $f_{i}(x) \neq 0$, we have by (3) and (4) that

$$
b(x)<\left(\left|f_{1}(x)\right|^{1 / 2}+\cdots+\left|f_{n}(x)\right|^{1 / 2}\right) / 2^{n}
$$

which contradicts the fact that

$$
b=\sum_{m}\left|f_{m}\right|^{1 / 2 / 2^{m}}
$$

Hence $f_{i}=0$ on $U$ for $1 \leq i \leq n$ and therefore $b=0$ on $U$ by (3). Thus $Z(I)=$ $\bigcap_{m} Z\left(f_{m}\right)=Z(b) \supset U$ which implies that $U=Z(I)$, that is, $Z(I)$ is an open set in $x$.

(b) implies (c). Suppose $Z(I)$ is open in $X$. Then $X-Z(I)$ is closed and hence compact so there exist $f_{1}, \cdots, f_{n} \in I$ with

$$
\left|f_{1}\right|+\cdots+\left|f_{n}\right| \geq \lambda>0
$$

on $X-Z(I)$. Let $f$ be the characteristic function of $X-Z(I)$. Then

$$
|f| \leq\left(\left|f_{1}\right|+\cdots+\left|f_{n}\right|\right) / \lambda
$$

and thus $f \in I$ since $\left(\left|f_{1}\right|+\cdots+\left|f_{n}\right|\right) / \lambda \in I$. Hence the vector lattice ideal $J$ generated by $f$ is contained in $I$. Moreover, if $b \in \mathrm{cl}(I)$, then $b=0$ on $Z(I)$ so $|b| \leq$ $\|b\| f$ and thus $b \in J$. Therefore $I$ is closed and generated by the idempotent $f$.

Corollary. If $X$ is connected, there are no proper closed countably generated vector lattice ideals in $C(X)$.

Proof. If $I$ is a closed countably generated vector lattice ideal in $C(X)$ where $X$ is connected, then $Z(I)=\varnothing$ or $Z(I)=X$ since $Z(I)$ is both open and closed in $X$. If $Z(I)=\varnothing$, then $I=C(X)$ while if $Z(I)=X, I$ is the zero ideal.

\section{BIBLIOGRAPHY}

1. L. Gillman, Countably generated ideals in rings of continuous functions, Proc. Amer. Math. Soc. 11 (1960), 660-666. MR 27 \#6120.

2. M. Stone, A generalized Weierstrass approximation theorem, Studies in Modern Analysis, Math. Assoc. of Amer. Studies in Math., vol. 1, Prentice-Hall, Englewood Cliffs, N. J., 1962. 